Original Research Paper

\title{
Time Dimension Parameters of the Dual-Porosity Reservoir Determination using Periodic Hydraulic Pulse Testing
}

\author{
Marat Ovchinnikov and Kushtanova Galiya Gatinishna
}

Department of Physics, Kazan Federal University, Russia

\author{
Article history \\ Received: 09-05-2016 \\ Revised: 07-09-2016 \\ Accepted: 08-09-2016 \\ Corresponding Author: \\ Marat Ovchinnikov \\ Department of Physics, Kazan \\ Federal University, Russia \\ Email: marov514@gmail.com
}

\begin{abstract}
The process of periodic hydraulic pulses propagation in porous fractured media of dual-porosity near a vertical well is considered. Using the calculation data as the base, it is shown that the effect of the time dimension constant on the form of the filtration wave curves is essential. The conclusions on possibility of hydrodynamic model types verification which adequately describe the filtration flows in considered media. The method for calculation of the dimensional time constants in equations for the non-stationary filtration is proposed.
\end{abstract}

Keywords: Filtration, Hydraulic Pulses, Fractured Porous Media of DualPorosity, Time Dimension Constants

\section{Introduction}

The dual porosity model (Barenblatt et al., 1960; Warren and Root, 1963) is commonly used to describe the filtration of fluids in various rock reservoirs of oil and gas with fractured porous structure. Under this model, porosity and permeability of the fracture and porous (block) reservoir spaces are introduced. Typically, the porosity of the fracture space $\left(m_{1}\right)$ is significantly smaller than the porosity of the block space $\left(m_{2}\right)$, while the permeability $\left(k_{1}\right)$ of the fracture space is substantially greater than the permeability of the block $\left(k_{2}\right)$ one.

For a description of non-stationary filtration of fluids in fractured porous media according to the continuum model, Barenblatt (1960) used the hypothesis of a linear relationship between the flow rate of fluid flow between pore and fissure subspaces:

$$
\begin{aligned}
& q=\alpha \frac{k_{2}}{\mu l^{2}}\left(P_{2}-P_{1}\right)=A\left(P_{2}-P_{1}\right) \\
& A=\alpha \frac{k_{2}}{\mu l^{2}}
\end{aligned}
$$

The model was later refined by Warren and Root (1963) by the introduction of the fracture and block spaces compressibility ( $\beta_{1}$ and $\beta_{2}$-fractures and porous compressibility):

$$
\bar{w}_{1}=-\frac{k_{1}}{\mu} \operatorname{grad} P_{1}
$$

$\bar{w}_{2}=0$

$$
\begin{aligned}
& \rho_{0} d i v \bar{w}_{1}+\frac{\partial\left(m_{1} \rho\right)}{\partial t}-q=0 \\
& \frac{\partial\left(m_{2} \rho\right)}{\partial t}+q=0 \\
& m_{1} \rho=m_{10} \rho_{0}+\rho_{0} \beta_{1} P_{1} \\
& m_{2} \rho=m_{20} \rho_{0}+\rho_{0} \beta_{2} P_{2}
\end{aligned}
$$

Here, indices 1-refers to fractures, 2-corresponds to blocks, 0 -corresponds to the initial parameter values, $k$ permeability, $m$-porosity, $w$-filtration velocity, $\mu$ viscosity, $\rho$-fluid density, $l$-linear block size, $\alpha$ dimensionless parameter order unit.

For the convenience of the unsteady filtration description the following constants dimension of time can be entered:

$$
\begin{aligned}
& \tau_{1}=\beta_{1} \frac{\mu l^{2}}{\alpha k_{2}}, \tau_{2}=\beta_{2} \frac{\mu l^{2}}{\alpha k_{2}}, \\
& \tau=\frac{\tau_{1} \tau_{2}}{\tau_{1}+\tau_{2}}, \tau_{1}, \tau_{2}>\tau
\end{aligned}
$$

The times $\tau_{1}, \tau_{2}$ introduced likewise include the elastic properties of rocks $\left(\beta_{1,2}\right)$, characteristic block linear sizes $(l)$, fluid viscosity $(\mu)$ and permeability $(k)$ of block space and are an important characteristic of the filtering process in fractured porous media. Thus, the knowledge of these times $\tau_{1}, \tau_{2}$ makes it possible to define 
characteristic linear size of fractured porous medium blocks. It is important for the understanding of the rock structure and the description of filtering in it. Evaluation of these times: at $\mu \sim^{10-1} \mathrm{~Pa} \cdot \mathrm{s}, k^{\sim^{10-14}} \mathrm{~m}^{2}, 1 \sim 1 \mathrm{~m}, \beta \sim^{10-9} \mathrm{~Pa}^{-1}$, $\alpha \sim 1, \tau \sim 10^{4} \mathrm{~s}$.

With regard to (1)-(9) linear model of fluid filtration in porous fractured reservoir can be rewritten. Barenblatt et al. (1960; Warren and Root, 1963; Van Golf-Racht, 1982) It can be presented as follows:

$\frac{1}{r} \frac{\partial}{\partial r}\left(r \frac{\partial}{\partial r}\left(P+\tau_{2} \frac{\partial P}{\partial t}\right)\right)=\frac{\partial}{\partial t}\left(P+\tau \frac{\partial P}{\partial t}\right)$

Here $\chi_{1}=k_{1} / \mu \beta$-fracture diffusivity coefficient. The issue is to find a solution for the radial flow near the vertical well with is $o$ tropic space in one-dimensional case of the filtration along the axis $r$ in a cylindrical coordinate system.

It obviously advantageous to determine the time constants $\tau_{1}, \tau_{2}$ for more accurate evaluation of the elastic characteristics of the pore spaces and cracks and, on the other side, for the correct determination of the reservoir filtration parameters $\sigma_{1}$ and the complex ratio $\chi_{1} / r^{2}$. In some cases we have to take into account the shape factors (Hassanzadeh et al., 2009).

The determination of filtration fractured porous media parameters $\sigma_{1}$ and the complex ratio $\chi_{1} / r^{2}{ }_{c}$ by pressure build-up test is a well-established procedure, but the interpretations of hydrodynamic experimental results in the natural conditions are difficult for their proper understanding. The difficulty is to define the time constants $\tau_{1}, \tau_{2}$. In these difficult cases, the immunity periodic pulse testing method outlined in (Buzinov and Umrihin, 1964; Johnson et al., 1966; Renner and Messar, 2006) can serve as an appropriate additional procedure combined to the standard build-up test. However, this method is used rarely (Nakao et al., 2005) and the procedures for the filtration parameters calculating need to be elaborated.

In the pulse testing method the periodic excited rate oscillations are created in some well and the registered response pressure oscillations are measured in the excited reactive well.

Suppose, the rate $q$ in a vertical well is created by a periodic change with frequency $\omega$ and phase shift $\delta_{q}$ and being expressed in the Fourier expansion form it is written as:

$$
q_{\text {total }}(t)=q_{0}+\sum_{n=1}^{n=1 \infty} q_{n} \cos \cos \left(\omega_{n} t+\delta_{q n}\right)
$$

Then the pressure $P$ will also change harmonically with the certain phase shift $\delta_{p}$ :

$$
P_{\text {total }}(t)=P_{0}+\sum_{n=1}^{n=1 \infty} P_{n} \cos \left(\omega_{n} t+\delta_{p n}\right)
$$

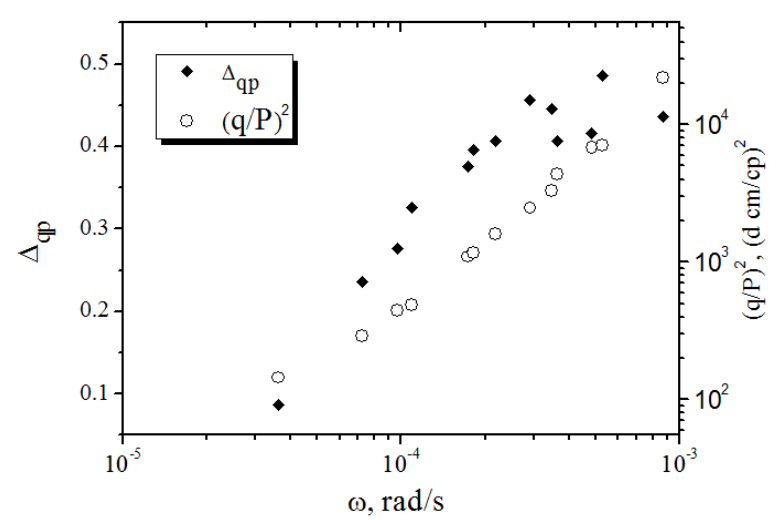

Fig. 1.The frequency dependence of the phase difference $\Delta_{q p}$ and the ratio $(q / P)^{2}$ (Molokovich et al., 2000)

Using the measured data of pressure and well rate at various times for each fixed frequency, one can calculate basic filtration parameters as transmissibility and hydraulic duffusivity constants. At further consideration, for convenience, we are going to consider only one harmonic oscillation mode of the rate and the pressure and omit the index $n$.

Figure 1 shows, as an example, the frequency dependences for the flow rate and pressure phase difference $\Delta_{q p}=\left(\delta_{q}-\delta_{p}\right)$ and the square of flow rate/pressure amplitudes ratio $(q / P)^{2}$ in the harmonically excited well (well number 4788, (Molokovich et al., 2000)) drilled in the fractured porous reservoir. These frequency dependences allow us to calculate, in particular, transmissibility of the fractured space $\sigma_{1}$ and the complex ratio $\chi_{1} / r_{c}^{2}$, where $\chi_{1}$-hydraulic diffusivity constant of fractured space, $r_{c}$ is well radius.

So, the aim of this work is to analyze the features of periodic pulses (waves) propagation in dual-porosity media and identify the time dimension parameters in the associated non-stationary filtration models.

\section{Methods}

The flow equation for the non-stationary filtration law in the dual-porosity fractured porous media is governed by Equation 3 (Molokovich et al., 2000) with4time dimension constants:

$$
\begin{aligned}
& \frac{\chi_{1} \tau_{1}}{r} \frac{\partial}{\partial r}\left[r \frac{\partial}{\partial r}\left(P+\left(\tau_{2}+\tau_{p}\right) \frac{\partial P}{\partial t}+\tau_{2} \tau_{p} \frac{\partial^{2} P}{\partial t^{2}}\right)\right]= \\
& \left(\tau_{1}+\tau_{2}\right) \frac{\partial P}{\partial t}+\left(\tau_{1} \tau_{2}+\tau_{1} \tau_{w}+\tau_{2} \tau_{w}\right) \frac{\partial^{2} P}{\partial t^{2}}+\tau_{1} \tau_{2} \tau_{w} \frac{\partial^{3} P}{\partial t^{3}}
\end{aligned}
$$

Here, $\tau_{1}, \tau_{2}$ are time dimension parameters characterizing the properties of fractures and blocks, respectively, $\tau_{p}$ and $\tau_{w}$-relaxation times. Making some assumptions that are needed to be specified, the authors 
of (Molokovich et al., 2000) evaluated the desired time constants experimentally: $\tau=6250 \mathrm{~s}, \tau_{2}=25000 \mathrm{~s}, \tau_{p}=$ $23094 \mathrm{~s}$ and $\tau_{w}=481 \mathrm{~s}$.

In this study, we consider a possibility to determine $\tau_{2}$ and $\tau$ parameters on the basis of experimental data associated with the periodic hydraulic pulse testing in terms of $(9,10)$.

The further development of this method implies(a) the realization of the conducting experiments at different frequencies, starting from $\omega \tau<<1$ to $\omega \tau \sim 1-10$; (b) the usage of the Fourier analysis data with simultaneous calculation of the corresponding amplitudes and phases of harmonics at different frequencies; (c) the calculation of the times $\tau_{2}$ and $\tau_{1}$.

The relationship between amplitudes and phases for the rate and pressure is defined earlier (Molokovich et al., 2000; Molokovich, 2006; Ovchinnikov, 2008) and expressed as:

$$
\begin{aligned}
& P \cos \cos \left(\omega t+\delta_{p 1}\right) \\
& =\frac{q}{2 \pi \sigma_{1}} \operatorname{Re}\left\{\frac{\operatorname{BesselK}\left(0, z_{c}\right)}{z_{c} \operatorname{BesselK}\left(1, z_{c}\right)} \exp \left(i t+i \delta_{q}\right)\right\} \\
& =\frac{q}{2 \pi \sigma_{1}} \operatorname{Re}\left\{(X+i Y) \exp \left(i t+i \delta_{q}\right)\right\}
\end{aligned}
$$

where, the complex argument of the Bessel functions (first kind of zero and first orders) is:

$$
\begin{aligned}
& z_{c}=\left|z_{c}\right| \exp \left(i\left(\frac{\pi}{4}+1 / 2\left(\operatorname{atan}(\omega \tau)-\operatorname{atan}\left(\omega \tau_{2}\right)\right)\right)\right), \\
& \left|z_{c}\right|=r_{c} \sqrt{\frac{\omega}{1}\left(\frac{1+\omega^{2} \tau^{2}}{1+\omega^{2} \tau_{2}^{2}}\right)^{1 / 4},} \\
& X=\operatorname{Re}\left\{\frac{\operatorname{Bessel} K\left(0, z_{c}\right)}{z_{c} \operatorname{Bessel} K\left(1, z_{c}\right)}\right\} . \\
& Y=\operatorname{Im}\left\{\frac{\operatorname{BesselK}\left(0, z_{c}\right)}{z_{c} \operatorname{Bessel} K\left(1, z_{c}\right)}\right\}
\end{aligned}
$$

From (15) we can define:

$$
\begin{aligned}
& 2 \pi \sigma_{1} \frac{P}{q}=\left(X^{2}+Y^{2}\right)^{1 / 2} \\
& \delta_{q}-\delta_{p}=\operatorname{atan}(Y / X)
\end{aligned}
$$

When $\left|z_{c}\right|<<1$, one can simplify expression for the pressure amplitude as:

$$
\begin{aligned}
& P=\frac{q}{2 \pi \sigma_{1}} \sqrt{\left(\ln \frac{\gamma\left|z_{c}\right|}{2}\right)^{2}+\Omega^{2}} \\
& \Omega^{2}=\left(\frac{\pi}{4}+\frac{1}{2}\left(\operatorname{atan}(\omega \tau)-\operatorname{atan}\left(\omega \tau_{2}\right)\right)\right)^{2}
\end{aligned}
$$

and the desired phase difference between the rate and the pressure in the well expressed as:

$$
\begin{aligned}
& \Delta_{q p}=\delta_{q}-\delta_{p}= \\
& =\operatorname{atan} \frac{\left(\frac{\pi}{4}+\frac{1}{2}\left(\operatorname{atan}(\omega \tau)-\operatorname{atan}\left(\omega \tau_{2}\right)\right)\right)}{\left|\ln \frac{\gamma\left|z_{c}\right|}{2}\right|} \\
& \Delta_{q p}^{0}=\delta_{q}-\delta_{p} \text { at } \tau_{2}=0, \tau=0, \\
& \Delta_{q p}^{\tau}=\delta_{q}-\delta_{p} \text { at } \tau_{2} \neq 0,
\end{aligned}
$$

Here, the parameter $\gamma=1.782 \ldots$ defines the Euler constant.

One can notice that the $\sigma_{1}$ transmissibility determines the linear relationship between the rate and pressure amplitudes, while the ratio $r_{c}^{2} / \chi_{1}$ and the time parameters $\tau_{2}$ and $\tau$ affecting the $q / P$ ratio and the phase difference between the rate and the pressure is described by the complicated relations. However, in the low-frequency limit, when $\omega \tau$ and $\omega \tau_{2}<<1$, the influence of $\tau_{2}$ and $\tau$ becomes insignificant and the corresponding parameters $\tau_{1}$ and $r_{c}{ }^{2} / \chi_{1}$ are determined from experiments as well as for models with $\tau_{2}$ and $\tau=$ 0 . Furthermore, we assume that these parameters are determined from the low-frequency experiments.

\section{Results}

Figure 2 and 3 show the calculated absolute values of rate/pressure ratio for the fixed transmissibility $\sigma_{1}$ and differences between rate and pressure phases at the various pulse frequencies for the cases: (a) $\tau_{2}=0, \tau=0$ and (b) $\tau_{2}=10^{4} \mathrm{~s}, \tau=2 \times 10^{3} \mathrm{~s}$. We see, if $\omega \tau>0.1$, the difference between the compared solutions of the Equation (10) with zero and non-zero values of the constants $\tau_{2}$ and $\tau$ becomes essential.

Here:

$$
\begin{aligned}
& \Delta_{q p}^{0}-\Delta_{q p}^{\tau}=\left(\delta_{q}-\delta_{p}\right)\left(a t \tau_{2}=0, \tau=0\right)- \\
& \left(\delta_{q}-\delta_{p}\right)\left(a t \tau_{2} \neq 0, \tau \neq 0\right)
\end{aligned}
$$

We see that the ratio of flow rate and pressure amplitudes differ for the cases of zero and non-zero values $\tau_{2}$ and $\tau$ (Fig. 2). In opposite to the case $\tau_{2}=0, \tau=0$, when the phase difference of the relationship between flow rate and pressure has the form of a monotone increasing function with respect to the increasing frequency, for nonzero values of $\tau_{2}$ and $\tau$ this phase difference is also determined and its frequency dependence has two local extrema: High and low (Fig. 3). It will be shown, how this fact is used to determine these times. 


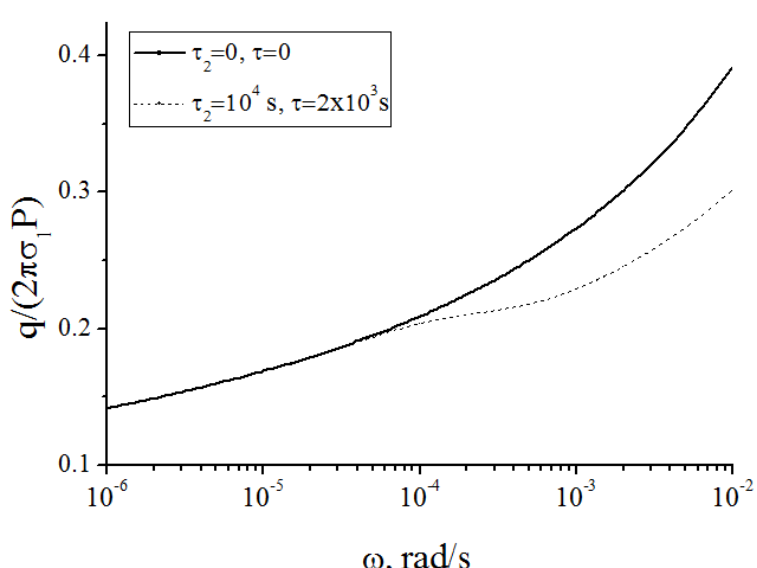

Fig. 2. Frequency dependence of the $q / 2 \pi \sigma_{1} P$ ratio at $r_{c}^{2} / \chi_{1}=1 \mathrm{~s}$

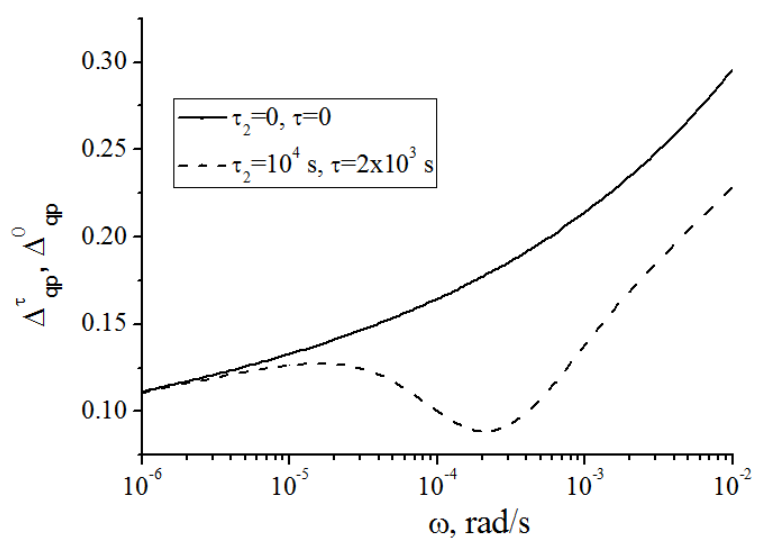

Fig. 3. Frequency dependence of the phase difference between the rate and the pressure at $r_{c}^{2} / \chi_{1}=1 \mathrm{~s}$

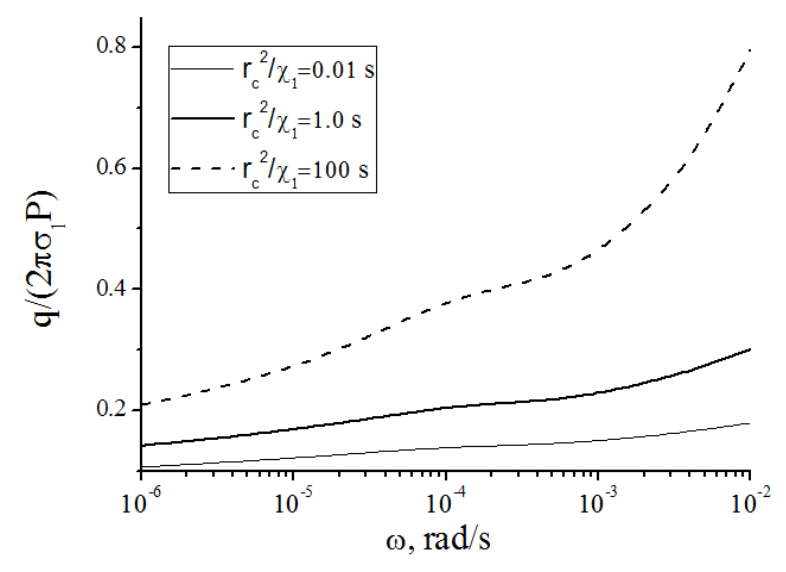

Fig. 4. Frequency dependence of the amplitude $q / 2 \pi \sigma_{1}$ Pratio at $\tau_{2}=10^{4} \mathrm{~s}, \tau=2 \times 10^{3} \mathrm{~s}$

Figure 4 and 5 show frequency dependences of the amplitude $q / P$ ratio and phase difference between the rate and pressure for the fixed values $\tau_{2}=10^{4}$ sand $\tau=2 \times 10^{3} \mathrm{~s}$, but at various $r_{c}{ }^{2} / \chi_{1}=0.01 \mathrm{~s}, r_{c}{ }^{2} / \chi_{1}=1 \mathrm{~s}, r_{c}{ }^{2} / \chi_{1}=100 \mathrm{~s}$.

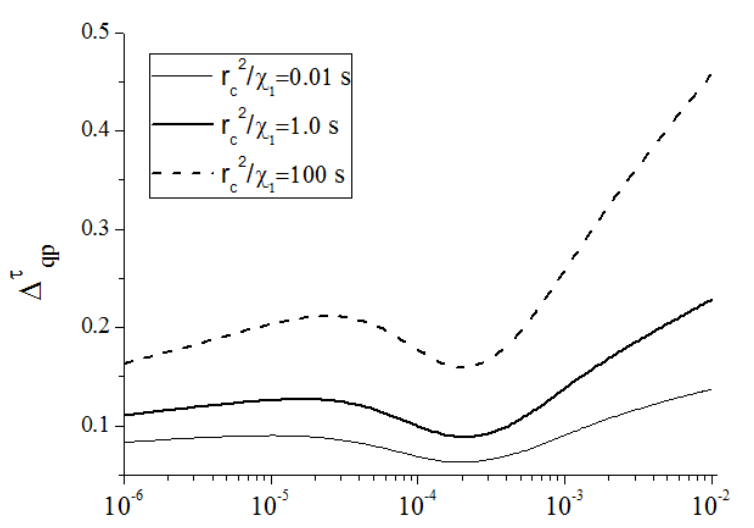

$\omega, \mathrm{rad} / \mathrm{s}$

Fig. 5. Frequency dependence of the phase difference between the rate and the pressure at $\tau_{2}=10_{4} \mathrm{~s}, \tau=2 \times 10^{3} \mathrm{~s}$

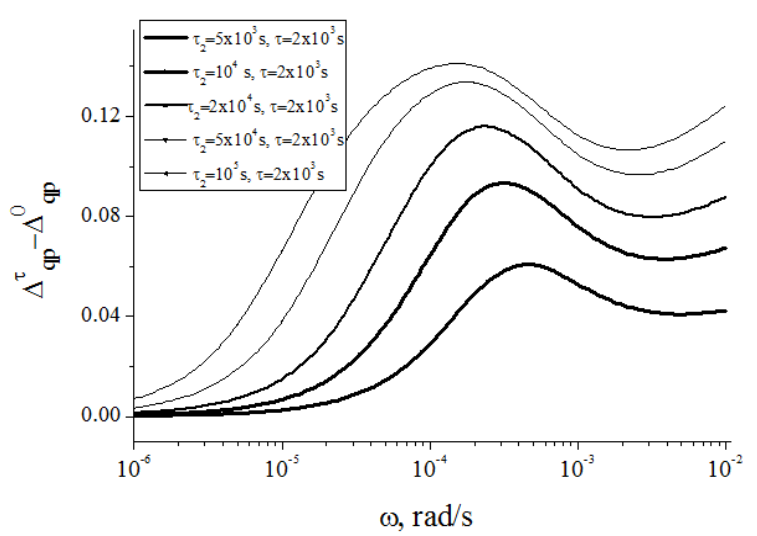

Fig. 6. Frequency dependence of the phase difference (13) at $\tau$ $=2 \times 10^{3}$ and $\tau_{2}=5 \times 10^{3}, 10^{4}, 2 \times 10^{4}, 5 \times 10^{4}, 10^{5} \mathrm{~s}$

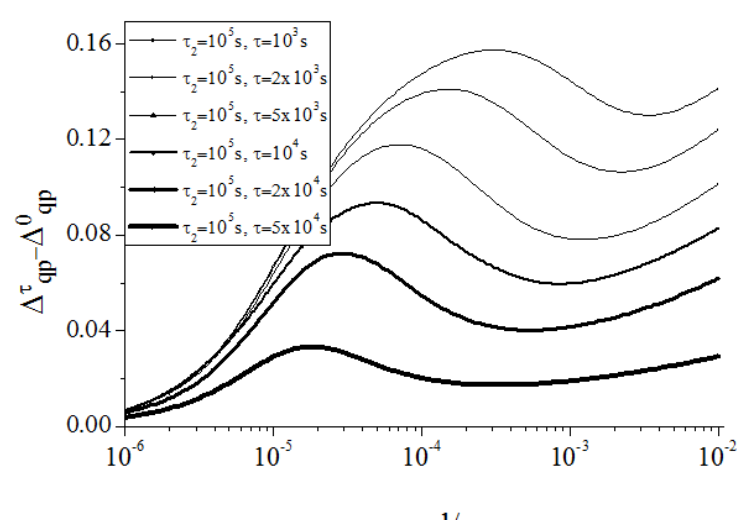

$\omega, \mathrm{rad} / \mathrm{s}$

Fig. 7. Frequency dependence of the phase difference (13) at $\tau_{2}$ $=10^{5}$ and $\tau=10^{3}, 2 \times 10^{3}, 5 \times 10^{3}, 10^{4}, 2 \times 10^{4}, 5 \times 10^{4} \mathrm{~s}$

We see that the changes of the parameter $r_{c}^{2} / \chi_{1}$ resulted in quantitative changes of the corresponding curves, though, qualitatively, the curves keep the same shape. 


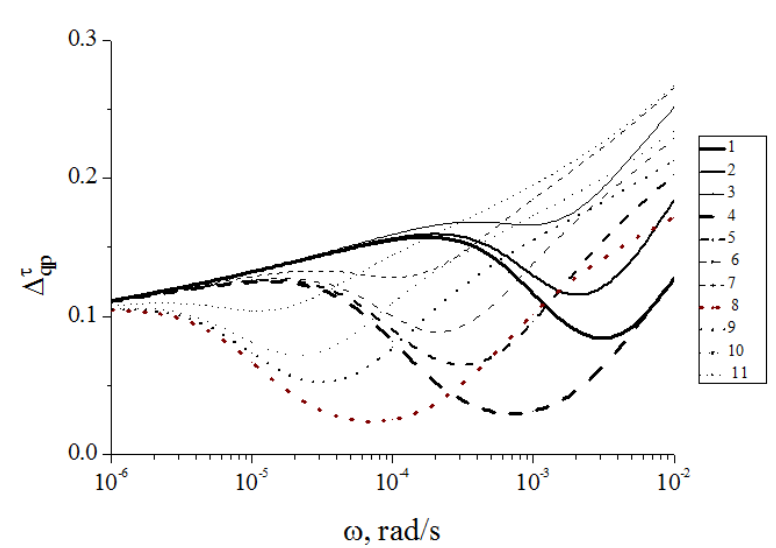

Fig. 8. The frequency dependence of the phase difference between the rate and the pressure at $1-\tau_{2}=10^{3} \mathrm{~s}, \tau=$ $10^{2} ; 2-\tau_{2}=10^{3} \mathrm{~s}, \tau=2 \times 10^{2} ; 3-\tau_{2}=10^{3} \mathrm{~s}, \tau=5 \times 10^{2} ; 4-$ $\tau_{2}=10^{4} \mathrm{~s}, \tau=2 \times 10^{2} \mathrm{~s} ; 5-\tau_{2}=10^{4} \mathrm{~s}, \tau=10^{3} \mathrm{~s} ; 6-\tau_{2}=10^{4}$ $\mathrm{s}, \tau=2 \times 10^{3} \mathrm{~s} ; 7-\tau_{2}=10^{4} \mathrm{~s}, \tau=5 \times 10^{3} \mathrm{~s} ; 8-\tau_{2}=10^{5} \mathrm{~s}, \tau=$ $2 \times 10^{3} \mathrm{~s} ; 9-\tau_{2}=10^{5} \mathrm{~s}, \tau=10^{4} \mathrm{~s} ; 10-\tau_{2}=10^{5} \mathrm{~s}, \tau=2 \times 10^{4}$ $\mathrm{s} ; 11-\tau_{2}=10^{5} \mathrm{~s}, \tau=5 \times 10^{4} \mathrm{~s} 1,2,3$-solid; 4,5,6,7-dash; $8,9,10,11-$ dot

The considered curves are divided into a some areas where the local minimum and maximum positions are determined by $\tau_{2}$ and $\tau$, while the angles of slope of the curves are governed by the ratio of these time values.

It presents an interest to consider the value of the phase difference between the cases with zero $\tau_{2}$ and $\tau$, as well as non-zero $\tau_{2}$ and $\tau$ for the fixed frequency:

$\tau_{2}-\tau \approx\left(\Delta_{q p}^{0}-\Delta_{q p}^{\tau}\right) \frac{2}{-}\left|\ln \left(\frac{\gamma}{2} r_{\mathrm{c}} \sqrt{\frac{\omega}{1}}\right)\right|$ at $\mathrm{w} \tau_{2}<0.3$

In Figures 6 and 7 we show the calculated differences between the rate and pressure phase for the cases of $\tau_{2}=$ $0, \tau=0$ and $\tau_{2} \neq 0, \tau \neq 0$. The calculations were performed for the values $r_{c}{ }^{2} / \chi_{1}=1$ sand transmissibility $\sigma_{1}=$ $1 \mathrm{~d} \mathrm{~cm} / \mathrm{cp}$.

We see that the dependence of the difference (21) of the frequency has a local maximum and minimum, their location and the corresponding values are determined by the values of the times $\tau_{2} \mathbf{\Lambda} \tau$.

\section{Discussion}

It is a difficult to find the exact analytical solution for the unknown parameters $\tau_{2}$ and $\tau$. However, the procedure of approximate calculation can be obtained from the analysis of experimental data.

One can notice that, in natural conditions, the time parameters $\tau_{2}$ and $\tau$ are located in the interval $10^{3}-10^{5} \mathrm{~s}$. By definition $\tau_{2} \geq \tau_{2}$ and, in practice, the corresponding ratio differs by 2-5 times. From Fig. 2-3 and 6-7, we see that with increase of frequency one can observe the following picture: Initially one can detect the parts depending on the time $\tau_{2}$, then, after the further increase of the frequency, other parts dependence on the time $\tau$ becomes significant.

Taking into account the results of periodical pulse experiment sat relatively low frequencies $\left(\omega \tau_{2}<0.3\right)$ and using formula (19), one can find very good approximation for evaluation of the difference between the values of $\tau_{2}$ and $\tau$ times in the form of:

$\tau_{2}-\approx\left(\Delta_{q p}^{0}-\Delta_{q p}^{\tau}\right) \frac{2}{-}\left|\ln \left(\frac{\gamma}{2} r_{\mathrm{c}} \sqrt{\frac{\omega}{1}}\right)\right|$

at $\mathrm{w} \tau_{2}<0.3$

Expression (22) is easily derived from (19) and (21) using a combination of the time values $\tau_{2}=0, \tau=0$ and $\tau_{2} \neq 0, \tau \neq 0$ and taking into account that for small values of the argument $\operatorname{atan}(\omega \tau) \approx \omega \tau$ with the value of the relative error less than 0.03 at $\omega \tau<0.3$ and $\ln \left(\frac{1+\omega^{2} \tau^{2}}{1+\omega^{2} \tau_{2}^{2}}\right)^{1 / 4}<0.02$ when $\omega \tau_{2}<0.3$. The unknown values $\sigma_{1}$ and complex ratio $\chi_{1} / r^{2}{ }_{c}$ are determined experimentally at low frequency of $\omega \tau_{2}<<1$.

In order to find the unknown $\tau_{2}$, one can use the fact that the phase differences $\Delta_{q p}^{0}-\Delta_{q p}^{\tau}(\omega)$ or $\Delta_{q p}^{\tau}$ have local extrema and their location are determined by the values of the times $\tau_{2}$ and $\tau$.

Actually, if one analyzes in detail the plots depicted in Fig. 3, 6, 7 or 8 at various values of the parameters $\tau_{2}$ and $\tau$, it is possible to derive the approximate formula for $\tau_{2}$ calculations in the conventional frequency range $10^{-6}<\omega<10^{-2} \mathrm{rad} / \mathrm{s}$.

For example, Figure 8 shows the values $\Delta_{q p}^{\tau}(\omega)$ for various $\tau_{2}$ and $\tau$ sets in semi-log scale for $r_{c}{ }^{2} / \chi_{1}=1 \mathrm{~s}$. Let $\omega=\omega_{\min }$ is the value of frequency at the local minimum and $\Delta_{\min }$ is the corresponding difference of the rate and pressure phases. Studying the set of points $\left\{\omega_{\min }, \Delta_{\min }\right\}$ for different values of the time $\tau_{2}$ and $\tau$, one can derive the following approximate formula for calculation of $\tau_{2}$ :

$\tau_{2} \approx \frac{10}{\omega_{\min }} 10^{-\frac{\Delta_{\min }}{0.03 \cdot \lg \left(\omega_{\min }\right)+0.255}}$

Using expressions (22) and (23), one can determine the time values $\tau_{2}$ and $\tau$, separately and, hence, the desired time $\tau_{1}$.

\section{Conclusion}

Determination of the filtration reservoir characteristics is an important task in the oil 
development and hydrogeology. The values of these parameters can be calculated according to the unsteady hydrodynamic experiments, such as pulse sequences. Since these calculations are a class of the ill-posed inverse problems of mathematical physics and the number of defined parameters increases with the complexity of the dynamic models used, it is desirable to obtain the required values of the parameters in the results of independent experiments. In this study, the authors propose, in addition to the standard methods, an original procedure of the multi-frequency probe reservoir. We propose a method for determination of the $\tau_{2}$ and $\tau$ time parameters, entering the theory of filtration in the fractured porous media and associated with elastic parameters of the cracked-block subspace. The method is based on the analysis of the characteristics of amplitude and phase-frequency characteristics of the harmonic filtration pressure waves. In accordance with new mathematical expressions obtained, it allows comparing the received full-scale study, depending on model and possibilities allowing evaluating the possible values of the time dimension parameters (relaxation times) for a fractured porous reservoir.

The formulas proposed by the authors are approximate but, nevertheless, they allow counting times $\tau_{2}$ and $\tau$ with accuracy of a few percent. The difference $\tau_{2}-\tau$, in the proposed method is defined at the frequency range of $0.1<\omega \tau<0.3$, which usually corresponds to the period of oscillations $\mathrm{T} \sim 10^{5}$. The approximate estimation of $\tau_{2}$ requires to be experiments performed at the higher frequencies $1<\omega \tau_{2}<10$. At the same time, the most of the experiments for the practical cases fall into a range $10^{-6}<\omega<10^{-2} \mathrm{rad} / \mathrm{s}$.

It should be noted that expressions written above are correct for the harmonic oscillations in the stationary state when the rate and pressure form a linear systems. When $\omega \tau<<1$ and $T>>\tau_{1}$ we deal with classical equation diffusion type and can determine $\varepsilon$ and $r_{c}{ }^{2} / \chi$ and, in the high-frequency limit $(\omega \tau>>1)$, we shall focus on overriding hydraulic duffusivity constant $\chi_{1}^{*}=\chi_{1}\left(\tau_{2} / \tau\right)$.

So, the detailed analysis of these curves allows to propose a method for evaluation of the time constants $\tau_{2}$ and $\tau$, which are important for understanding the filtration processes in fractured porous media.

\section{Acknowledgement}

We wish to acknowledge prof. M. Khairullin for useful discussions.

\section{Author Contributions}

Ovchinnikov Marat: Wrote the article as a leading author, formula, analysis and results interpretation.

Kushtanova Galiya: Programming and calculation.

\section{Ethics}

This article is original and contains unpublished material. The corresponding author confirms that the other author has read and approved the manuscript and no ethical issues involved.

\section{References}

Barenblatt, G.I. and P. Zeltov Yu, 1960. Basic flow equations for homogeneous fluids in naturally fractured rocks. Doclady Akademii Nauk SSSR, 132: $545-548$.

Buzinov, S.N. and D. UmrihinI, 1964. The Study of Wells and Reservoirs at Elastic Filtration Condition. 1st Edn., Nedra, Moscow, pp: 273.

Hassanzadeh, H., M. Pooladi-Darvish and S. Atabay, 2009. Shape factor in the drawdown solution for well testing of dual-porosity systems. Adv. Water Resources, 32: 1652-1663.

DOI: $10.1016 /$ j.advwatres.2009.08.006

Johnson, C., R. Greenkorn and E. Woods, 1966. PulseTesting: A new method for describing reservoir flow properties between wells. J. Petroleum Technology, 18: 1599-1604.

Molokovich, Y.M., 2006. Nonequilibrium Filteration and its Application in the Oil Field Practice. 1st Edn., Regular Andchaotic Dynamics, MoscowIzhevsk, pp: 214.

Molokovich, Y.M., A.I. Markov and G.G. Kushtanova, 2000. Development of Fractured Porosity Reservoir Unsteady Drainage. 1st Edn., Publishing House Regent, Kazan, pp: 156.

Nakao, S., T. Ishido, K. Hatakeyama and K. Ariki, 2005. Analysis of pulse tests in a fractured geothermal reservoir-a case study at the Sumikawa field in Japan. Proceedings of the World Geothermal Congress, Apr. 24-29, Antalya, Turkey, pp: 24-29.

Ovchinnikov, M.N. and A.Y. Zavidonov, 2008. Searching for an optimum frequency. Geore. Sources, 1: 21-22.

Renner, J. and M. Messar, 2006. Periodic pumping tests. Geophys. J. Int., 167: 479-493.

DOI: $10.1111 /$ j.1365-246X.2006.02984.x

Van Golf-Racht, T.D., 1982. Fundamentals of fractured reservoir engineering. Elsevier, Amsterdam, ISBN-10: 0080868665, pp: 732.

Warren, J.E. and P.J. Root, 1963. The behavior of naturally fractured reservoirs. Society Petro. Eng. J., 3: 245-255. 